This item was submitted to Loughborough's Research Repository by the author.

Items in Figshare are protected by copyright, with all rights reserved, unless otherwise indicated.

\title{
Physical principles of the amplification of electromagnetic radiation due to negative electron masses in a semiconductor superlattice
}

\section{PLEASE CITE THE PUBLISHED VERSION}

http://dx.doi.org/10.1134/S002136401424014X

\section{PUBLISHER}

Springer (@ Pleiades Publishing)

\section{VERSION}

AM (Accepted Manuscript)

\section{PUBLISHER STATEMENT}

This work is made available according to the conditions of the Creative Commons Attribution-NonCommercialNoDerivatives 4.0 International (CC BY-NC-ND 4.0) licence. Full details of this licence are available at: https://creativecommons.org/licenses/by-nc-nd/4.0/

\section{LICENCE}

CC BY-NC-ND 4.0

\section{REPOSITORY RECORD}

Shorokhov, Aleksei V., Nikolai A. Pyataev, Nikolai N. Khvastunov, Timo Hyart, F.V. Kusmartsev, and Kirill N. Alekseev. 2019. "Physical Principles of the Amplification of Electromagnetic Radiation Due to Negative Electron Masses in a Semiconductor Superlattice". figshare. https://hdl.handle.net/2134/26111. 


\title{
Physical Principles of the Amplification of Electromagnetic Radiation Due to Negative Electron Masses in a Semiconductor Superlattice
}

\author{
A. V. Shorokhov and M. A. Pyataev \\ Mordovian National Research University, Saransk 430005, Russian Federation \\ N. N. Khvastunov \\ Mordovian State Pedagogical Institute, Saransk 43000\%, Russian Federation \\ T. Hyart \\ Department of Physics, University of Jyväskylä, Jyväskylä FI-40014, Finland \\ F. V. Kusmartsev and K. N. Alekseev \\ Department of Physics, Loughborough University, Loughborough LE11 3TU, United Kingdom
}

\begin{abstract}
In a superlattice placed in crossed electric and magnetic fields, under certain conditions, the inversion of electron population can appear at which the average energy of electrons is above the middle of the miniband and the effective mass of the electron is negative. This is the implementation of the negative effective mass amplifier and generator (NEMAG) in the superlattice. It can result in the amplification and generation of terahertz radiation even in the absence of negative differential conductivity.
\end{abstract}

PACS numbers: 67.57.Lm, 76.60.-k

The idea of using the effect of negative effective mass of charge carriers in semiconductors for creating negative effective mass amplifier and generator (NEMAG) was proposed by H. Krömer [1] in 1958 by an example of holes with a negative effective mass in germanium placed in an electric field. It was experimentally implemented for the first time in the mid-1980s [2]. The experimental and theoretical works in this field were reviewed in [3]. Conditions for the realization of the NEMAG become particularly favorable when a bulk semiconductor is additionally placed in a static magnetic field [4].

In this work, we show that NEMAG can also be implemented in a semiconductor superlattice operating in a miniband transport regime when it is placed in crossed electric and magnetic fields of moderate strengths. In this case, under certain conditions, a population inversion can arise at which the average energy of electrons is above the middle of the miniband and the effective electron mass becomes negative in average. This in turn results in the amplification of terahertz radiation in the absence of instabilities caused by negative differential conductivity and being characteristic of the classical amplification schemes [5, 6]. In contrast to quantum cascade structures of weakly coupled wells, where the achievement of an inverse population between Landau levels allows the creation of a tunable quantum generator of terahertz radiation [7], the dynamics of electrons in the strongly coupled superlattice is in essence semiclassical [8, 9]. Note that terahertz electroluminescence in the $\mathrm{SiC}$ structure having a natural superlattice was recently observed in [10]. This indicates the possibility of generating terahertz ra-

* alex.shorokhov@mail.ru diation in superlattice structures operating in miniband transport regime.

The minimal semiclassical model of the superlattice in crossed fields was proposed by Polyanovskii [1]. He analytically found the current and its dependence on the field strengths. As follows from the results reported in [12, 13], this simple single miniband model satisfactorily describes the experimental currentvoltage characteristics. Various electronic and optical properties of superlattices, demonstrating the single miniband transport regime under the action of electric and magnetic fields of various configurations, were studied in [14]-[21]. Finally, the significant wideband amplification of terahertz waves in a superlattice placed in crossed fields was recently predicted in 22. on the basis of numerical analysis.

However, the physical principles of amplification in such a system can hardly be revealed by numerical analysis alone. The understanding of these principles is not only of fundamental interest but also necessary for the choice of the optimal parameters of an amplifier. In this work, we combine the analytical [11] and numerical 222] methods to demonstrate the feasibility of NEMAG in the superlattice and to clarify its relation to the previously described terahertz amplification regime.

The analysis of electron trajectories in the phase space makes it possible to visually understand the physical essence of the effect. For this reason, we first consider the ballistic transport regime for electrons in the superlattice placed in a static electric field $\mathbf{E}$, which is directed along the axis of the superlattice ( $x$ axis), and a static magnetic field $\mathbf{H}$, which is directed along the $z$ axis. In the tight-binding approximation, the dependence of the 
energy $\varepsilon$ on the quasimomentum $\mathbf{p}$ has the standard form

$$
\varepsilon(\mathbf{p})=\frac{\Delta}{2}\left(1-\cos \frac{p_{x} d}{\hbar}\right)+\frac{1}{2 m_{\perp}}\left(p_{y}^{2}+p_{z}^{2}\right),
$$

where $\Delta$ is the width of the miniband, $d$ is the period of the superlattice, and $m_{\perp}$ is the effective mass of the electron along the layers of the superlattice. Using the equation of motion

$$
\dot{\mathbf{p}}=e \mathbf{E}+\frac{e}{c} \mathbf{V} \times \mathbf{H}
$$

and the standard formula for the electron velocity, $\mathbf{V}=$ $\partial \varepsilon(\mathbf{p}) / \partial \mathbf{p}$, we obtain the following semiclassical equations describing the ballistic transport regime:

$$
\left\{\begin{array}{l}
\dot{K}_{x}=\omega_{B}+\omega_{c} K_{y} \\
\dot{K}_{y}=-\omega_{c} \sin K_{x}
\end{array}\right.
$$

Here, $K_{x}=p_{x} d / \hbar, K_{y}=\sqrt{m_{0} / m_{\perp}} p_{y} d / \hbar, \omega_{B}=e E d / \hbar$ is the Bloch frequency, $m_{0}=2 \hbar^{2} / \Delta d^{2}$ is the effective electron mass at the bottom of the miniband along the axis of the superlattice, and $\omega_{c}=e H / \sqrt{m_{x} m_{\perp}} c$ is the effective cyclotron frequency. The momentum along the $z$ axis is conserved.

According to Eq. (3), the projection of the quasimomentum on the $x$ axis, which governs the motion of electrons along the superlattice axis, satisfies the pendulum equation

$$
\ddot{K}_{x}+\omega_{c}^{2} \sin K_{x}=0 .
$$

We note that the electric field enters into this equation only through the initial conditions. The amplification of external radiation in this system is possible only in the nonlinear regime, because this equation at small $K_{x}$ values is a usual equation of small oscillations that allows only resonant (cyclotron) absorption of radiation, rather than amplification.

From Eq. (4) we obtain the following equation of a phase trajectory in the quasimomentum space:

$$
\left(\frac{\omega_{B}}{\omega_{c}}+K_{y}\right)^{2}+4 \sin ^{2}\left(\frac{K_{x}}{2}\right)=\left(\frac{\Omega}{\omega_{c}}\right)^{2},
$$

where $\Omega$ is determined by the initial conditions for the quasimomentum, $K_{x}(0)=K_{x}^{0}$ and $K_{y}(0)=K_{y}^{0}$, and by the electric and magnetic field strengths.

In the general case, the frequency of nonlinear oscillations has the form 11.

$$
\Omega_{e f f}=\left\{\begin{array}{l}
\frac{\pi \omega_{c}}{2 \mathbf{K}\left(\Omega / 2 \omega_{c}\right)}, \Omega<2 \omega_{c} \\
\frac{\pi \Omega}{2 \mathbf{K}\left(2 \omega_{c} / \Omega\right)}, \Omega>2 \omega_{c}
\end{array}\right.
$$

where $\mathbf{K}(x)$ is the complete elliptic integral of the first kind. We note that such oscillations have been observed experimentally [9].

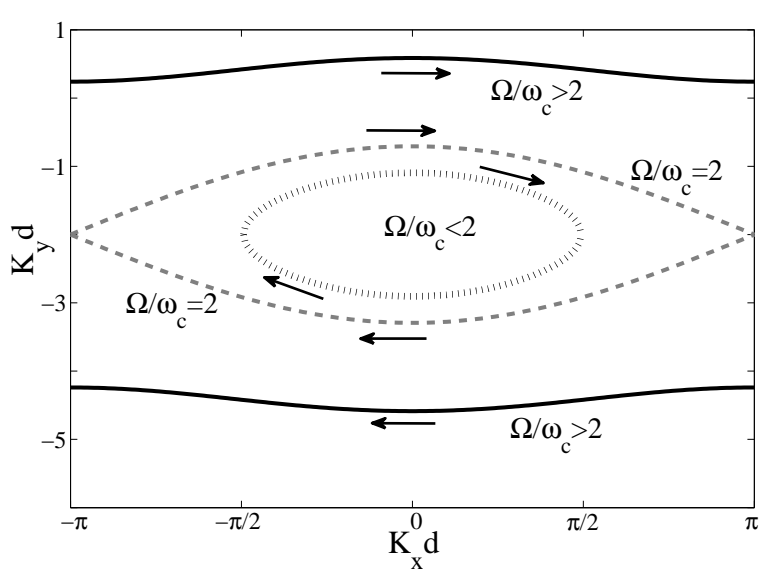

FIG. 1: Ballistic trajectories in the $\left(K_{x} ; K_{y}\right)$ plane.

We now indicate some characteristic features of ballistic trajectories of electrons, which can be conveniently analyzed by considering trajectories in the quasimomentum space (Fig. 1).

The equation of the pendulum (44) has a separatrix $2 \omega_{c}=\Omega$ separating physically different regimes of the periodic motion of electrons. No oscillations exist on the separatrix. Here electrons only asymptotically approach the top of the miniband. However, this trajectory is unstable against any noise or fluctuations. For $2 \omega_{c}>\Omega$, the magnetic field is strong enough to confine electrons in the first Brillouin zone, and quasi-cyclotron oscillations occur. With an increase in the magnetic field, trajectories become more similar to usual cyclotron trajectories. In this case, electrons oscillate near the bottom of the miniband. For $\Omega / \omega_{c}>2$, electrons undergo the complete motion over the miniband, which is accompanied by Bragg reflections. The frequency of Bloch oscillations is modified owing to the magnetic-field-induced bend of trajectories. It is noteworthy that the frequencies of both quasi-Bloch and quasi-cyclotron oscillations decrease upon approaching the separatrix. The choice of a certain type of motion of electrons strongly depends on their initial quasimomentum. In a particular case of zero initial conditions $\left(K_{x}(0)=0, K_{y}(0)=0\right)$ the equation of the pendulum has the separatrix at $2 \omega_{c}=\omega_{B}$. These zero initial conditions correspond to the case of low temperatures and low electron densities, where only states near the bottom of the miniband are occupied in equilibrium. This case will be considered in what follows.

If the scattering of electrons is taken into account within the approximation of constant relaxation time $\tau$, the separatrix at low temperatures separates the regions of the positive and negative slopes in the currentvoltage characteristic of the superlattice [11, 22] (with allowance for the shift of the maximum by a value of about $\tau^{-1}$, characteristic of dissipative systems). This is quite clear because the electron undergoing quasi-cyclotron oscillations does not reach the edge of the Brillouin zone and does not undergo total reflection. Thus, the genera- 
tion of high-frequency radiation without negative differential conductivity is in principle possible only for quasicyclotron oscillations, which correspond to the oscillational rather than rotational regime of pendulum (4).

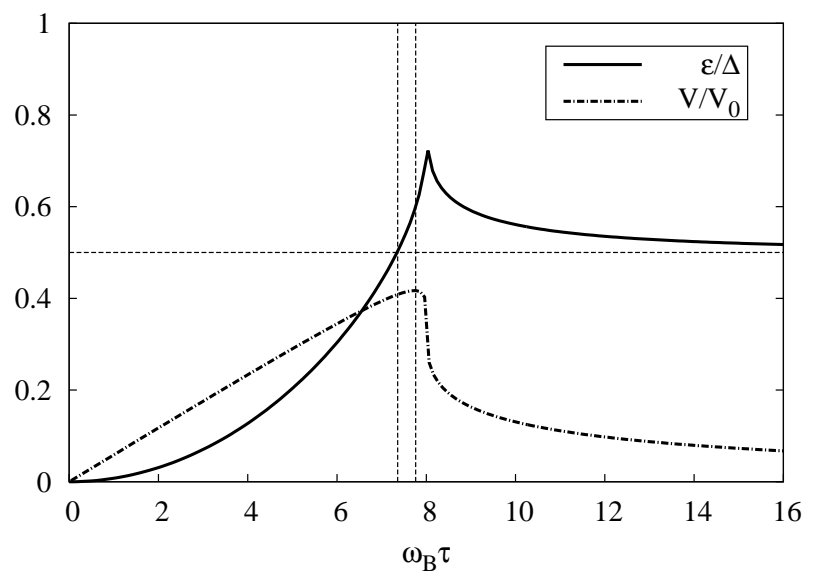

FIG. 2: Average energy (solid line) and drift velocity (dash-dotted line) versus the scaled electric field $\omega_{B} \tau$ at $\omega_{c} \tau=4$. The velocity is normalized to the maximum miniband velocity of the electron $V_{0}=\Delta d / 2 \hbar$ and the energy is normalized to $\Delta$. The horizontal dashed straight line corresponds to the middle of the miniband and the vertical dashed straight lines correspond to

Bloch frequencies at which the effective mass and derivative of the velocity with respect to $\omega_{B}$ change sign.

In physical terms, the possibility of the amplification of radiation is due to a specific behavior of the average energy and effective mass. We calculate the average energy of electrons along the $x$ axis under the assumption that they are on the bottom of the miniband at the initial time:

$$
\left\langle\varepsilon_{x}\right\rangle=\frac{1}{\tau} \int_{0}^{\infty} \varepsilon_{x}(t) \mathrm{e}^{-t / \tau} d t
$$

Here,

$$
\varepsilon_{x}=\frac{\Delta}{2}\left[1-\cos \frac{p_{x} d}{\hbar}\right]=\Delta \operatorname{sn}^{2}\left(\frac{\omega_{B} t}{2} ; \frac{2 \omega_{c}}{\omega_{B}}\right),
$$

where $\operatorname{sn}(u ; k)$ is the elliptic Jacobi function.

Then, at $\omega_{B} / \omega_{c}>2$, we obtain

$$
\begin{aligned}
\left\langle\varepsilon_{x}\right\rangle & =\frac{2 \pi^{2} \Delta}{k^{2} \mathbf{K}^{2}(k)} \sum_{n, l=0}^{\infty} \frac{q^{n+l+1}}{\left(1-q^{2 n+1}\right)\left(1-q^{2 l+1}\right)} \\
& \times\left[\frac{1}{1+\left[(n-l) \pi \omega_{B} \tau / 2 \mathbf{K}(k)\right]^{2}}-\right. \\
& \left.-\frac{1}{1+\left[(n+l+1) \pi \omega_{B} \tau / 2 \mathbf{K}(k)\right]^{2}}\right]
\end{aligned}
$$

Here, $k=2 \omega_{c} / \omega_{B}$ and $q=\mathrm{e}^{-\pi \mathbf{K}^{\prime} / \mathbf{K}}$, where $\mathbf{K}^{\prime}$ is the complete elliptic integral of the first kind of the argument $\sqrt{1-k^{2}}$. According to Eq. (9) and Fig. 2, the average energy in the limit $\omega_{B} \rightarrow \infty$ tends to the middle of the band $\left(\left\langle\varepsilon_{x}\right\rangle \rightarrow \Delta / 2\right)$, which is typical of conventional Bloch oscillations.

The average energy at $\omega_{B} / \omega_{c}<2$ has the form

$$
\begin{aligned}
\left\langle\varepsilon_{x}\right\rangle & =\frac{2 \pi^{2} \Delta}{\mathbf{K}^{2}} \sum_{n, l=0}^{\infty} \frac{q^{n+l+1}}{\left(1-q^{2 n+1}\right)\left(1-q^{2 l+1}\right)} \\
& \times\left[\frac{1}{1+\left[(n-l) \pi \omega_{c} \tau / \mathbf{K}\right]^{2}}\right. \\
& \left.-\frac{1}{1+\left[(n+l+1) \pi \omega_{c} \tau / \mathbf{K}\right]^{2}}\right],
\end{aligned}
$$

where $k=\omega_{B} / 2 \omega_{c}$ in contrast to Eq. (9).

At $\omega_{B}=2 \omega_{c}$, we obtain

$$
\begin{aligned}
\left\langle\varepsilon_{x}\right\rangle & =-3+\frac{2}{\omega_{B} \tau}\left[\psi\left(\frac{\omega_{\mathrm{B}} \tau+1}{2 \omega_{\mathrm{B}} \tau}\right)-\psi\left(\frac{1}{2 \omega_{\mathrm{B}} \tau}\right)\right] \\
& +4 \sum_{n, s=1}^{+\infty} \frac{(-1)^{n+s}}{1+(n+s) \omega_{B} \tau},
\end{aligned}
$$

where $\psi(x)$ is the Euler psi function.

The possibility of amplifying high-frequency radiation in the superlattice without magnetic field is traditionally attributed to the use of the negative differential conductivity regime, which corresponds to the condition $\omega_{B} \tau>1$ [5]. The magnetic field not only shifts the maximum of the currentvoltage characteristic of the superlattice toward stronger fields [12] but also leads to the appearance of a new region of amplification on the left of the peak of the currentvoltage characteristic, as well as to a significant increase in the magnitude of amplification at negative differential conductivity 22. . We believe that these amplification effects can be explained within the concept of negative effective mass. The strongest amplification should be expected when the average energy of electrons is above the middle of the miniband and the effective mass becomes negative, as in the NEMAG case, because according to the formula 23]

$$
m_{x}\left(\varepsilon_{x}\right)=\frac{m_{0}}{1-2 \varepsilon_{x} / \Delta},
$$

the transition to population inversion is directly related to the appearance of negative effective mass.

Figure 2 shows the dependences of the average energy of electrons, given by Eqs. (9-11), and their drift velocity [11] on the normalized electric field $\omega_{B} \tau$. When the electron gas is heated, electrons acquire energy. In conditions of negative differential conductivity and at least for long superlattices with ohmic contacts, this energy will be spent on the formation of propagating high-field domains owing to the development of charge instabilities [24]. However, when the working point is chosen in the segment of the current-voltage characteristic with a 
positive slope, instabilities are absent and the collected energy can be directly transformed to radiation. The maximum of the energy coincides with the point of transition from rotational to oscillational regime $\left(\omega_{B}=2 \omega_{c}\right)$, whereas the maximum of the average velocity is shifted leftward from this point by a value of $\tau^{-1}$. Such a behavior of the energy and velocity is well known in the theory of oscillations: Amplitude resonance is always shifted from energy resonance by a value of about the damping coefficient. It can be clearly seen in the figure that the region where the average electron energy becomes above the middle of the band begins with field values lower than the value corresponding to the peak of the currentvoltage characteristic. Therefore, there exists a region near the separatrix where the average energy of the electron is above the middle of the band, whereas the slope of the currentvoltage characteristic is positive and, consequently, the system is stable. Such a system operates as a classical analog of quantum generators of radiation.

The above statements are confirmed by the numerical analysis of the absorption of the probe field $E(t)=$ $E_{\omega} \cos (\omega t)$, directed along the axis of the superlattice. The amplitude of the probe field $E_{\omega}$ is small and the frequency $\omega$ varies in a quite wide range. In this case, it is assumed that the value of $\omega$ in experiments is governed by an external resonant circuit. To calculate the absorption coefficient, we used the path-integral method for solving the Boltzmann equation for the superlattice [15, 22]. For the numerical analysis, we used the following typical parameters of the GaAs superlattice: $d=6 \mathrm{~nm}$, $\Delta=60 \mathrm{meV}$, density of free carriers $n=10^{16} \mathrm{~cm}^{-3}$, and dielectric constant $\varepsilon=13$.

Figure $3 \mathrm{a}$ shows the region in the parametric space $\left(\omega_{B} \tau, \omega \tau\right)$ that corresponds to the amplification of stimulated radiation. As can be seen in the figure, the amplification region is wider than the region of negative effective mass. This can serve as an additional illustration of a statement that negative effective mass is only one of the conditions for the appearance of amplification [25][27]. It is obvious that not all frequencies are amplified identically. In particular, the maximum possible gain is reached at the frequencies $\omega_{m}$ shown in the same figure. As was mentioned above, amplification in this case is directly attributed to oscillations of electrons in the miniband. Indeed, it is seen in Fig. 3a that the dependence of $\omega_{m}$ on the electric field strongly correlates with the corresponding dependence for the effective frequency of electron oscillations $\Omega_{\text {eff }}$.

Figure $3 \mathrm{~b}$ shows the dependence of the gain on $\omega_{B} \tau$ for the frequencies $\omega$ at which the gain is maximal (i.e., for $\left.\omega_{m}\right)$. The maximum gain is reached near the separatrix and at the relatively low frequencies. As was previously emphasized in [22], the magnitude of gain is very large near the separatrix. We now estimate the range of frequencies $\omega$. Taking a value of about 200 fs for the relaxation time of the typical superlattice, we conclude that the amplified frequencies are in the range from 500 $\mathrm{GHz}$ to several terahertz. The necessary magnetic field
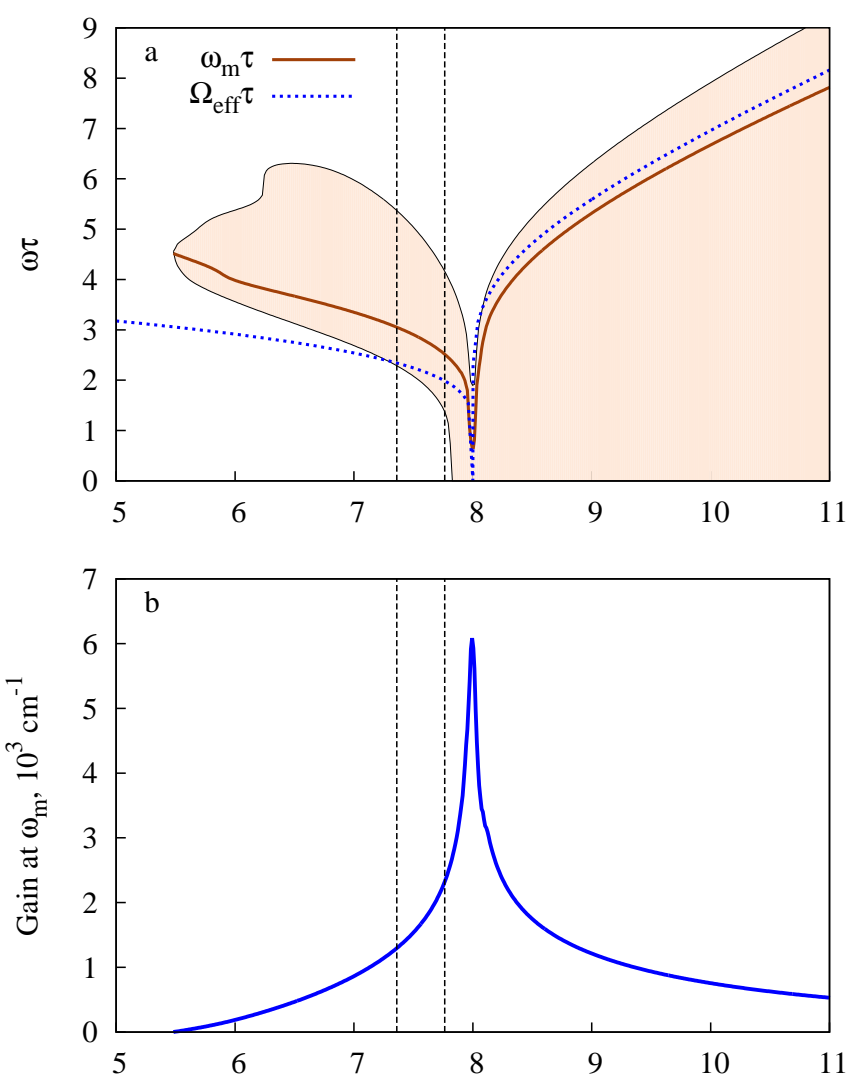

FIG. 3: (a) Region of fields and frequencies corresponding to the amplification of a weak alternating field in the superlattice (shaded area). The solid line is

the plot of frequencies $\omega_{m} \tau$ at which the gain is maximal and the dashed line is the plot of effective frequencies $\Omega_{\text {eff }} \tau$ of oscillations of ballistic electrons. (b) Maximum possible gain versus the scaled electric field field $\omega_{B} \tau$ at $\omega_{c} \tau=4$. The meaning of the vertical dashed straight lines is the same as in Fig. 2.

strength should be several tesla.

To conclude, we note that negative effective mass of miniband electrons in superlattices can also appear due to the application of either static electric field at conventional Bloch oscillations [5, 23] or ac pump field at parametric amplification 28] and other parametric effects [29, 30]. However, in both indicated cases, the mass averaged over the period of oscillations is positive and, therefore, no population inversion exists. The application of a perpendicular magnetic field makes electron oscillations nonlinear and the average mass of electrons negative in the strong nonlinearity regime. In this respect, we also would like to attract attention to the problem of terahertz radiation amplification in the superlattice placed in a tilted magnetic field [22]. Here miniband electrons demonstrate strongly nonlinear dynamics which is manifested in the existence of a chaotic web [16]. Possibility of contribution of the negative effective mass in the amplification in this situation is a very interesting problem 
for further investigations.

We are thankful to N. S. Prudskikh for technical assistance. This work was supported by Ministry of Education and Science of Russian Federation (project no. 2.2665.2014), Engineering and Physical Sciences Research Council (grant no. EP/I01490X/1), Royal Society (UK-India Collaboration).
[1] H. Krömer, Phys. Rev. 109, 1856 (1958).

[2] A. A. Andronov, A. M. Belyantsev, V. I. Gavrilenko, E. P. Dodin, Z. F. Krasil'nik, V. V. Nikonorov, and S. A. Pavlov, JETP Lett. 40, 989 (1984).

[3] A. A. Andronov, Sov. Phys. Semicond. 21, 701 (1987).

[4] A. A. Andronov, A. M. Belyantsev, V. I. Gavrilenko, E. P. Dodin, Z. F. Krasil'nik, V. V. Nikonorov, S. A. Pavlov, and M. M. Shvarts, Sov. Phys. JETP 63, 211 (1986).

[5] L. Esaki, R. Tsu, IBM J. Res. Dev. 14, 61 (1970).

[6] S. A. Ktitorov, G. S. Simin, and V. Ya. Sindalovskii, Sov. Phys. Solid State 13, 1872 (1971).

[7] F. R. Jasnot, L.-A. de Vaulchier, Y. Guldner, G. Bastard, A. Vasanelli, C. Manquest, C. Sirtori, M. Beck, and J. Faist, Appl. Phys. Lett. 100, 102103 (2012).

[8] A. Ya. Shik, Sov. Phys. Semicond. 7, 187 (1973).

[9] T. Bauer, J. Kolb, A. B. Hummel, H. G. Roskos, Y. Kosevich, and K. Köhler, Phys. Rev. Lett. 88, 086801 (2002).

[10] V. I. Sankin, A. V. Andrianov, A. O. Zakhar'in, and A. G. Petrov, JETP Lett. 94, 362 (2011).

[11] V. M. Polyanovskii, Sov. Phys. Semicond. 14, 718 (1980).

[12] A. Sibille, J. F. Palmier, A. Celeste, J. C. Portal, and F. Mollot, Europhys. Lett. 13, 279 (1990).

[13] J. F. Palmier, A. Sibille, G. Etemadi, A. Celeste, and J. C. Portal, Semicond. Sci. Technol. 7, B283 (1992).

[14] F. G. Bass, V. A. Lykakh, and A. P. Tetervov, Sov. Phys. Semicond. 14, 1372 (1980).

[15] F. G. Bass, V. V. Zorchenko, and V. I. Shashora, Sov. Phys. Semicond. 15, 263 (1981).

[16] T. M. Fromhold, A. A. Krokhin, C. R. Tench, S. Bujkiewicz, P. B. Wilkinson, F. W. Sheard, and L. Eaves,
Phys. Rev. Lett. 87, 046803 (2001).

[17] T. M. Fromhold, A. Patane, S. Bujkiewicz, P.B. Wilkinson, D. Fowler, D. Sherwood, S. P. Stapleton, A.A. Krokhin, L. Eaves, M. Henini, N. S. Sankeshwar, and F. W. Sheard, Nature 428, 726 (2004).

[18] Yu. A. Kosevich, A. B. Hummel, H. G. Roskos, and K. Köhler, Phys. Rev. Lett. 96, 137403 (2006).

[19] T. Feil, C. Gerl, and W. Wegscheider, Phys. Rev. B 73, 125301 (2006).

[20] N. V. Demarina, E. Mohler, A. Lisauskas, H. G. Roskos, Phys. Rev. B 80, 24530 (2009).

[21] A. O. Selskii, A. A. Koronovskii, A. E. Hramov, O. I. Moskalenko, K. N. Alekseev, M. T. Greenaway, F. Wang, T. M. Fromhold, and A. G. Balanov, Phys. Rev. B 84, 235311 (2011).

[22] T. Hyart, J. Mattas, and K. N. Alekseev, Phys. Rev. Lett. 103, 117401 (2009).

[23] A. A. Ignatov, K. F. Renk, and E. P. Dodin, Phys. Rev. Lett. 70, 1996 (1993).

[24] N. Alexeeva, M. T. Greenaway, A. G. Balanov, O. Makarovsky, A. Patane, M. B. Gaifullin, F. Kusmartsev, and T. M. Fromhold, Phys. Rev. Lett. 109, 024102 (2012).

[25] P. Kaus, Phys. Rev. Lett. 3, 20 (1959).

[26] S. Rodriguez, Phys. Rev. 115, 821 (1959).

[27] C. Kittel, Proc. Nat. Acad. Sci. USA 45, 744 (1959).

[28] T. Hyart, A. Shorokhov, and K. N. Alekseev, Phys. Rev. Lett. 98, 220404 (2007).

[29] Yu.A. Romanov and Yu.Yu. Romanova, J. Exp. Theor. Phys. 91, 1033 (2000).

[30] A.V. Shorokhov, N.N. Khvastunov, T. Hyart, and K.N. Alekseev, J. Exp. Theor. Phys. 111, 822 (2010). 